\title{
The Integrated Disaster Reduction Intelligent Service System and its Application
}

\author{
Jiping Liu ${ }^{\text {a }}$, Yong Wang a , Mengmeng Liu ${ }^{\mathrm{a}, \mathrm{b},}$ *, Shenghua Xu ${ }^{\text {a }}$, Tao Jiang ${ }^{\mathrm{a}, \mathrm{b}}, \mathrm{Yang} \mathrm{Gu}^{\mathrm{a}, \mathrm{c}}$ \\ ${ }^{a}$ Chinese Academy of Surveying and Mapping; No. 28 Lianhuachi West Road, Haidian District, Beijing100830, China; \\ ${ }^{b}$ Liaoning Technical University; No.28 Yulong Road, Xihe District, Fuxin123000, China; e-mail: liuflame123@gmail.com; \\ ${ }^{c}$ Huaihai Institute of Technology; No. 59 Cangwu Road, Haizhou District, Lianyungang, China; \\ * Corresponding author
}

\begin{abstract}
Geo-spatial information technology can provide data resources, positioning benchmarks, basic framework and key technologies for disaster prevention and reduction. At present, there are some problems in China's disaster reduction services. Too much focus is placed on decision-making but not on early warning. In addition, the integration of technology, system and application of disaster reduction services is not enough. There is still a lack of a unified understanding an integrated disaster reduction intelligent service system. In order to provide technical support for China's comprehensive disaster reduction decision analysis, from the perspective of surveying, mapping, and geoinformation, this paper introduces an integrated disaster reduction intelligent service prototype system, which is concluded key technologies such as indoor and outdoor integrated emergency location, multi-source emergency data fusion, disaster scene visualization, and disaster model analysis services. Moreover, the integrated disaster reduction intelligent service prototype system has been applied in relevant emergency departments in Tibet and Xinjiang, realizing the integrated sensing, positioning, integration, analysis and service of emergency information.
\end{abstract}

Keywords: Disaster Reduction, GIS, The Integrated Disaster Reduction Intelligent Services

\section{Introduction}

China is one of the countries which seriously affected by natural disasters in the world. According to statistics for the past 5-20 years, in every year, there are 3573.5 people died in the earthquake in China, and the average annual economic losses were 29.587 billion yuan. The annual average number of deaths caused by geological disasters such as landslides, collapses, and mudslides were 1,090, so economic losses were between 12 and 15 billion yuan; The annual death toll from floods and disasters was 1647.8 people, and economic losses were 110.634 billion yuan (Fan et al., 2017; Zhu et al., 2014; Annelli, 2006). Affected by the coupling of natural, economic and social factors such as global climate change, extreme weather events, and their secondary disasters will increase in the future. Destructive earthquakes are still frequent occurrences. The suddenness of natural disasters, abnormalities and complexity have increased. At the same time, there are some problems in China's disaster reduction services. Too much emphasis is placed on decision-making and not on early warning. The integrated disaster reduction services system is incomplete. Local governments at all levels have weakly integrated disaster prevention and reduction capabilities (Faraj and Xiao, 2006; Martinis et al., 2015; Ajmar et al., 2015).

At present, the comprehensive disaster reduction "smart" technology solves the problem of predictable, controllable and preventable emergency disaster events, which has been the consensus views of experts in related research fields at home and abroad. In recent decades, comprehensive disaster reduction "smart" system has been applied and verified in a few developed countries or regions. The National Incident Management System (NIMS) of the United States was established in 2008. NIMS adopts the life-cycle model to realize cross-section and multi-event emergency response management, and guides all departments, governments at all levels, nongovernmental organizations and private institutions to cooperation (Gasparini et al., 2007; Allen et al., 2009). The Copernicus Emergency Management Service (EMS) provides timely and accurate geospatial information services for natural disasters, man-made emergencies and humanitarian crises in the EU (Jang and Suh, 2010). The earthquake and tsunami warning system covering the whole country established by Japan has built disaster information system, emergency response support system and early evaluation system (Yu et al., 2017). U-City plan of South Korea is a redefinition of the future high-tech city, which uses IT infrastructure, technology and service integration to provide intelligent urban services for urban housing, urban economy and urban transportation. And, of course, the U - Facility Management (FM) can not only provide urban infrastructure resources configuration and efficient use of services, but also it can be more important to provide very flexible resource scheduling services in the emergency response process (Liu et al., 2017; Zhang et al., 2017).

This paper deeply explores the key technologies of integrated disaster reduction intelligent services, including multi-source data integration, emergency plan management and intelligent matching technology, 
emergency space model analysis technology, knowledge map and emergency active push service technology, etc.

This paper aims to solve the key technologies of the integrated disaster reduction services system about an integrated perception, positioning, fusion and integrated scene simulation of emergency information such as disaster events, various disaster bodies and pregnant environments, as well as knowledge-based disaster reduction services. This paper takes six kinds of disasters such as earthquake, flood, geological disaster, urban fire, traffic accident and building collapse as research objects. We build an integrated disaster reduction intelligent service system by adopting a loosely coupled system architecture system based on micro-service under a unified standard. This system realizes the positioning of all kinds of heterogeneous systems and services integrated emergency equipment, real-time access and storage of sensing data, integration of related resources, and enhanced visualization of 3D disaster scene by combining situational awareness and cross-media integrated display technology. And it realizes three-dimensional space calibration and linkage visualization of multi-type emergency equipment, rescue personnel and major scenes. For emergency rescue decision-making, command and scheduling, information release, provide support for full space positioning, heterogeneous fusion, scene visualization, model calculation and deduction of the basis of comprehensive disaster reduction intelligent service architecture. At the same time, it can quickly customize different emergency disaster reduction applications according to different business needs, meet the needs of different industries, regions and departments, and provide rapid functional customization for the development of practical applications.

\section{Key Technologies of the Integrated Disaster Reduction Intelligent Service System}

Under the guidance of geographic information science and public security science, integrate BeiDou network, communication network, Internet and sensor network. A variety of information carriers, breakthrough highprecision indoor and outdoor integration seamless positioning, take into account for the semantic, and scale of time and space characteristics of disaster and multiscale spatial information integration, large-scale complex disaster scene dynamic modeling, simulation, and enhance the visual, for comprehensive disaster reduction Multiscale cross-media dynamic mapping, integrated disaster model construction and other key technologies, and establish a comprehensive process model for integrated disaster reduction intelligent services.

\subsection{High-Precision Indoor and Outdoor Integration Seamless Positioning}

High-precision indoor and outdoor positioning technology requires real-time acquisition of static and dynamic highprecision positioning data in complex environments. therefore, put forward the multi-source signal indoor highprecision positioning technology, research and analysis under complicated indoor environment more source of indoor positioning technology, key research based on Wi-
Fi, bluetooth fingerprint acquisition, update, and fast fingerprint matching rapid positioning computation technology, solution based on smartphones, tablets and other common terminal equipment real-time access to the problem of high accuracy indoor location information; Research on high-precision indoor positioning technology based on Bluetooth and Wi-Fi wireless signals, comprehensively use GNSS/UWB/INS/LiDAR sensors to synchronize various sensors in time and space. On this basis, data fusion of different sensor data is carried out according to different environments, and an indoor and outdoor integrated seamless positioning technology based on GNSS/UWB/INS/LIDAR is proposed.

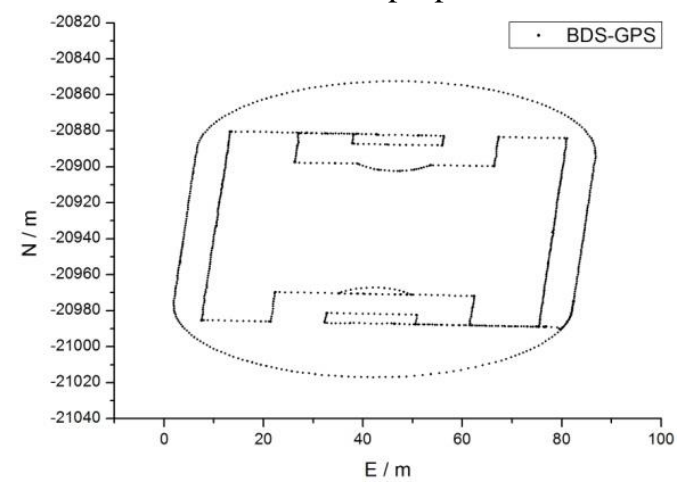

Figure 1. BDS/GPS dynamic positioning results

\subsection{Multi-Source Disaster and Multi-Scale Spatial Information Integration}

Multi-source disaster and multi-scale spatial information integration technology mainly solves the association method of high-precision matching mapping between disaster information and geographical entity, including the research content of spatial information extraction and spatial matching technology of disaster information The deep integration method of polymorphic disaster information considering semantic relationship, mainly researches video stream geocoding, GNSS geographic information data parsing, extracting video key frames and key frame positive correction, offline extraction of key frames; scene space target multi-feature tracking processing technology The multi-objective feature tracking and recognition method based on image and image data is studied. The multi-feature recognition such as SIFT feature, texture feature and structural feature is used to realize the recognition and localization of the target object (Qiu et al., 2017). Based on multi-feature tracking and recognition, combined with data modeling method. The function of model positioning and model assembly of the target in the basic geographic information data is realized, which is supported by the multi-state information fusion technology of indoor and outdoor scenes.

\subsection{Fusion and Enhanced Visualization of Large-Scale and Complex Disaster Scene}

In view of at the demand of disaster emergency command and judgment, the dynamic fusion modeling method of disaster scene, the visualization method of disaster information based on Symbolization and realistic coordination, and the technology of disaster threedimensional scene construction and network service 
publishing are studied. An adaptive network threedimensional visualization method based on WebGL technology for diverse terminals is proposed. From the perspective of users, taking the network environment accessed by device terminals, terminal performance, realtime visualization frame rate of browsers and other parameters as influencing factors, the key technologies such as diversified organization of $3 \mathrm{~d}$ data, adaptive quadtree organization and dynamic index construction, adaptive scheduling of $3 \mathrm{~d}$ scene and so on are mainly studied, and an adaptive $3 \mathrm{~d}$ scene construction model under the comprehensive influence of multiple influencing factors is established (Guo and Ying, 2017; Li et al., 2017). Considering the future needs of disaster-oriented virtual reality/augmented reality interactive visualization, Kinect Fusion algorithm is adopted to realize the rapid reconstruction of the real world surface model, and then the constructed virtual disaster scene is placed on the reconstructed object surface, so as to realize the complete fitting between the virtual object and the real world model, improve the lifelike immersion of user experience.

\subsection{Integrated Disaster Model Analysis and Comprehensive Service}

Spatial analysis and prediction models of six typical disasters, such as earthquake, flood, geological hazard, urban fire, traffic accident and building collapse are studied. Semantic-driven model service framework technology is constructed to realize the standardization, dynamic and semantic collaborative use of multi-hazard model and provide model analysis support for integrated disaster reduction services. Due to the massive mass, heterogeneity and complex relationship of disaster information, multi-source data grid management technology is proposed to construct the ontology of earthquake, flood, geological hazard, urban fire, traffic accident and building collapse. Relevant knowledge in the respective fields of the six types of disasters, providing a common understanding of the knowledge in the field, and proposing an active push service based on the emergency disaster knowledge map (Wang et al., 2016). An emergency case library matching method based on nearest neighbor algorithm is proposed. The intelligent matching and online negotiation of emergency plan are solved by using the plot reproduction and collaborative editing concurrent control strategy technology.

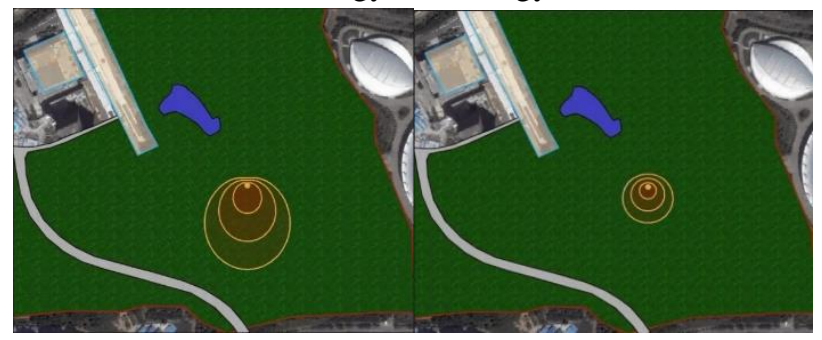

Figure 2. Calculation model of fire model under different wind speed parameters

\section{Construction of the Integrated Disaster Reduction Intelligent Service System}

Focusing on six types of disasters, such as earthquake, flood, geological hazard, urban fire, traffic accident, building collapse, etc., and considering the process of predisaster prevention, pre-disaster warning and rescue, postdisaster reconstruction and evaluation, serving the central government and other relevant departments in decisionmaking, disaster site command and rescue personnel, the people and disaster victims, Combining the key technologies of adaptive visualization, model deduction, map matching and large data stream computing, it provides personalized and multi-granularity Comprehensive disaster reduction intelligent services for decision makers, commanders and the people (Zhang et al., 2015). The logical architecture design is divided into five basic levels: user layer, service layer, system layer, support layer, and data layer, as shown in the following figure.

\section{(1) Data Layer}

The data layer is the integration of comprehensive disaster reduction intelligent service system of data and resource base. It is an important medium for comprehensive disaster reduction information storage, including basic topographic data, DEM digital elevation model, thematic geographic information data, image data, tile data, dynamic positioning data and other spatial data, as well as nonspatial data such as statistical database, thematic database and multimedia database.

\section{(2) Support Layer}

The support layer is the technical support of the integrated disaster reduction intelligent service system. $\mathrm{t}$ mainly carries out secondary development through cloud GIS engine, GIS analysis component, UCMap and other GIS development components based on GIS path analysis, buffer analysis and other functions, based on mining technology, data such as disaster model data, disaster visualization data, and disaster maps are extracted and stored to provide a data foundation for the service layer.

\section{(3) System Layer}

The system is a secondary development based on GIS development components such as cloud GIS engine, GIS analysis component, UCMap etc. The system's geographic information service includes massive data storage, visualization, disaster analysis, disaster information access, model deduction, information push, etc. Through the integration, deduction, display and other processing of spatial and non-spatial geographic information data, it can provide users with a variety of intelligent analysis and services: situational awareness information access, dynamic calibration of rescue equipment, multidimensional linkage display of multiple situation information at the disaster scene, intelligent matching of emergency plans, disaster model deduction, disaster site emergency command, multi-expert online consultation, disaster information intelligent push, etc.

\section{(4) Service Layer}

The service layer is the user interaction layer of the integrated disaster reduction intelligent service system. It provides dynamic calibration, disaster information retrieval, emergency response intelligent matching, emergency command, online negotiation and other personalized comprehensive disaster reduction for 
different people such as leadership decision-making, emergency command, ordinary people, etc.

\section{(5) User Layer}

The service targets of the integrated disaster reduction intelligent service system are divided into three audiences: decision makers, commanders and ordinary people. The three different groups of the central and related departments leading decision-making, disaster scene command and rescue personnel, ordinary people and disaster victims have different views on disaster information. The system provides personalized multigranular comprehensive disaster reduction intelligent services for different groups of people.

The integrated disaster reduction intelligent service system builds five modules: disaster information management and database construction, emergency event location and perception, emergency information extraction and statistics, emergency model analysis and deduction, comprehensive disaster reduction intelligent service, which is based on the system architecture.

(1) The disaster information management and database construction module contains basic information such as population and economy and legal person, emergency special information such as emergency support resources and protection targets and hazards, emergency history information and related disaster information of Internet mining. Finally, an emergency knowledge base is formed by integrating various types of emergency information, extracting multi-level features and analyzing multi-level associations and other processes.
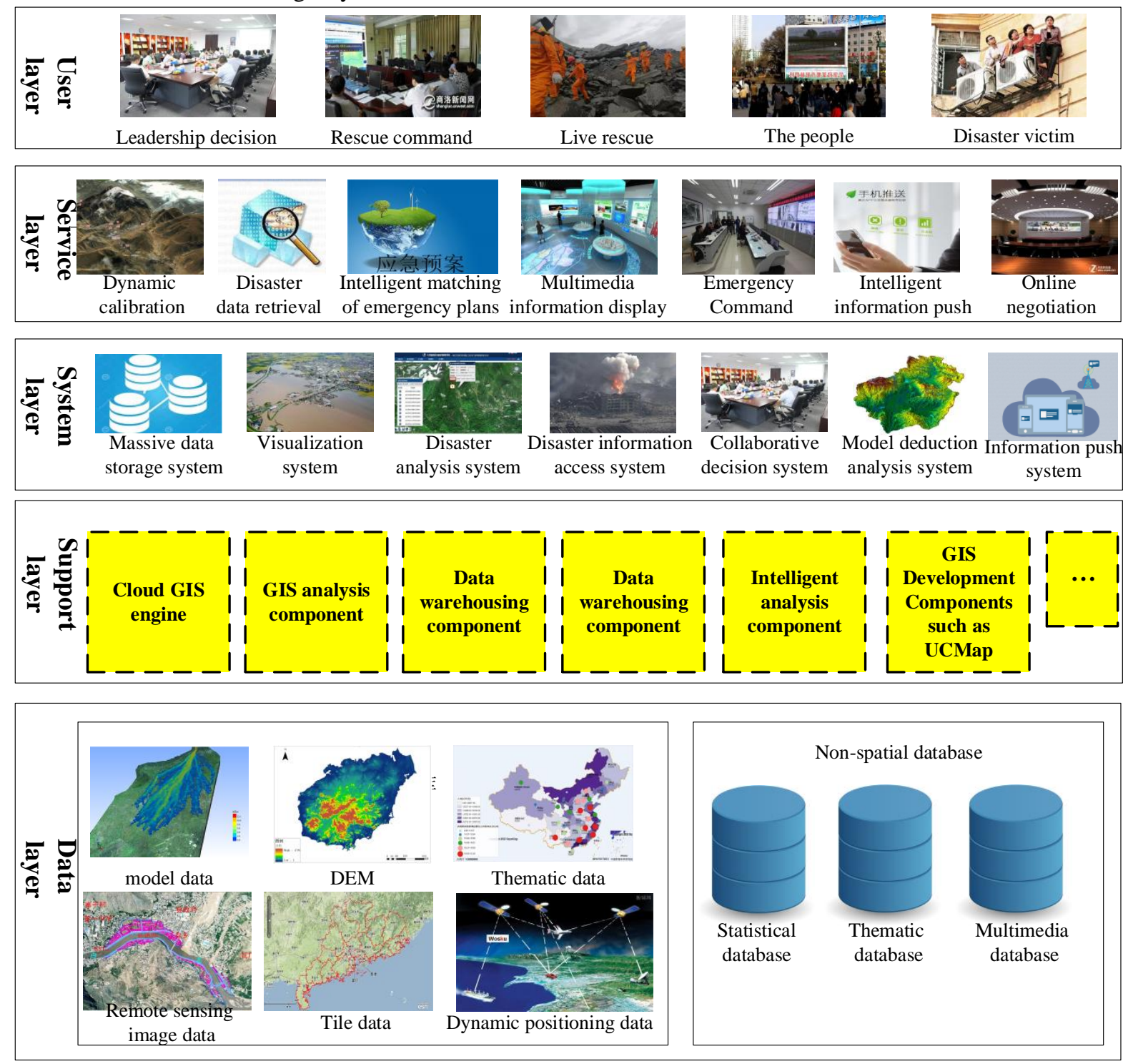

Figure 3. The Architecture of the Integrated Disaster Reduction Intelligent Service System

(2) The emergency event location and perception module includes functions such as disaster event location, integrated indoor and outdoor location of the disaster site, and intervention of disaster site perception information.
And it has realized real-time positioning and 3D scene visualization of personnel, vehicles and relief materials at the disaster site. 
(3) The emergency information extraction and statistics module mainly includes disaster topic information extraction, graphic range information query, and emergency topic information query. Also, it has realized the functions of extracting, integrating, correlating and statistical analysis of various types of disaster information within the scope of disasters.

(4) The emergency model analysis and deduction module not only realizes general spatial analysis models such as personnel evacuation, 2-3-dimensional path analysis, earthwork calculation, spatial superposition analysis, and buffer analysis, but also includes professional emergency disaster reduction models and 2-3-dimensional deductive analysis services for typical disasters such as fires, earthquakes, traffic accidents, and geological disasters.

(5) The comprehensive disaster reduction intelligent service module includes comprehensive mapping services, knowledge map services, intelligent plan services, and active push services. It has realized emergency rapid adaptive mapping service, typical disaster knowledge mapping service, emergency notification push service, etc, so it can automatically generate an integrated disaster reduction intelligent emergency plan.

\section{Application of the Integrated Disaster Reduction Intelligent Service System}

In order to satisfy the demand of maintaining stability and dealing with emergencies, we integrate the research results of the project to develop application system of integrated disaster reduction intelligent service of emergencies in Tibet and the system of maintaining stability in Xinjiang on the basis of emergency GIS platform by integrating the interfaces of the system. And then, we load basic geographic information data and the topical data to provide comprehensive services for maintaining stability.

\subsection{Application System of the Integrated Disaster Reduction Intelligent Service of Emergencies in Tibet}

The project of emergency geographic information platform of Tibet autonomous region is necessary for government planning, economic and social development, construction of major projects, environmental protection, emergency handling and disaster prevention and reduction, and It is also an urgent need to accelerate the process of regional information, realize the sharing of regional geographic information resources and reduce redundant construction.

The functions of the demonstration application are basic geographic information of Tibet, emergency event management, major emergency event disposal, temple information, basic emergency information and geological disaster information, etc. The part of basic geographic information includes introduction of Tibet, geographical name retrieval, route planning and road control. The part of emergency event management can create and retrieve events. The part of emergency thematic information includes important target data, name query, shape query and other functions, and important target data includes roads, bridges, schools and temples. The part of major emergency event disposal lists corresponding events, types and disposal report.

The part of temple information includes temple information, name query and shape query. The part of basic emergency information includes population statistics, geographical condition data, 1:500 data of five cities and two regions, management of mobile targets, and overall emergency plan for public emergencies in Tibet. The part of geological disaster information includes risk prediction and analysis of geological hazards and prevention materials for counties with frequent occurrence of geological hazards.

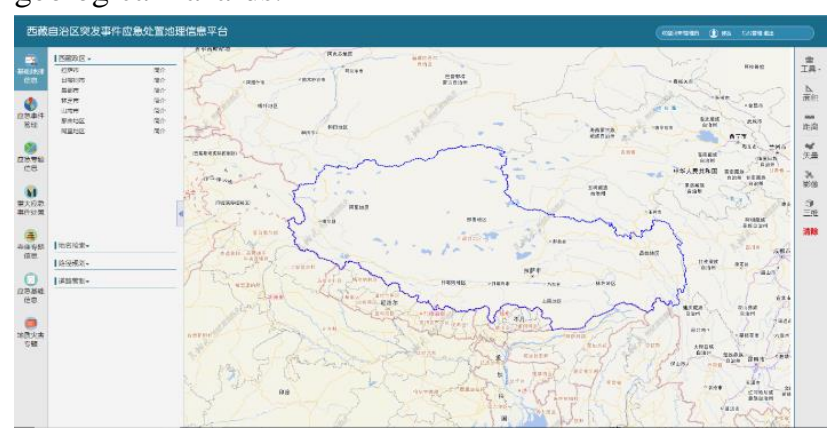

Figure 4. Function interface of geographic information platform for emergency response in Tibet autonomous region

We build the geographic information service platform for emergency response and upgrade the existing platform; The platform could realize geographic information sharing, spatial analysis, emergency thematic information loading, and emergency event mapping, retrieval, statistics, etc. We also can improve the management system of thematic geographic information data for emergencies in the autonomous region; At last, we provide government departments with convenient, fast and detailed emergency response geographic information services.

\subsection{Demonstration Application of the Integrated Disaster Reduction Intelligent Service in Xinjiang}

Driven by the current goal of social development and longterm stability in Xinjiang, its business needs are growing stronger and stronger. The emergency platform is no longer able to meet the needs of the new business, lack of accurate positioning of spatial locations for emergency events and rapid acquisition and processing of event site information. Unable to analyse ethnic minority distribution areas and simultaneous warning and disposal, Unsatisfied emergency dispatch, Unable to report to the Coordinating Commander and Information Liaison Group at the first time. In response to the local needs of Xinjiang, Xinjiang's comprehensive and comprehensive disaster reduction intelligent services have emerged.

The main features of the demonstration application: high precision indoor and outdoor positioning vintegrated intelligent analysis 、 intelligent emergency plan 、 intelligent modular drawing. Includes real-time positioning sensor information display, situation analysis, short message communication: video access function: ability to realize time-space geographic information of emergency scenes, on-site real-time collection and organic 
combination, visual display: spatial analysis functions such as buffer analysis.

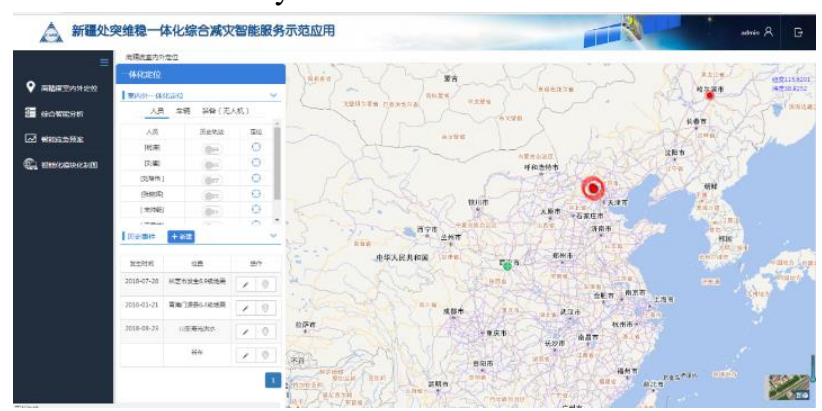

Figure 5. Demonstration application of comprehensive disaster reduction intelligent service in Xinjiang

Xinjiang's emergency and integrated disaster reduction intelligent service system integrates with Xinjiang's emergency management department's existing stability and information system. Develop SOA-based architecture、 $\mathrm{B} / \mathrm{S}$ service model, establish a location-based stability database, build an integrated and comprehensive intelligent service department to implement a system of stability and stability and form a comprehensive emergency response system with comprehensive coverage, sensitive touch and rapid response. Emergency response to achieve stability and stability in Xinjiang, intelligent assistant analysis, command and dispatch of the whole process of scientific and information, as well as multisectoral and inter-regional emergency response and collaborative operations, the actual needs of Xinjiang are urgently stabilized. On the basis of the emergency platform, Combining the interface support of the integrated disaster reduction intelligent service system, developing the demonstration application system of Xinjiang's sudden maintenance service, build a comprehensive coverage, touch sensitive, rapid response emergency response system, improve the emergency platform of the autonomous region, realize the emergency response mechanism of scientific and informational command and dispatch and multi-departmental and inter-regional emergency linkage and collaborative operations.

\section{Conclusion}

Disaster reduction service is an important part of emergency mapping and disaster research, from the perspective of surveying and mapping, so that the integrated intelligent service system for disaster reduction this paper introduced is deeply studied and discussed. Based on the research summary of disaster reduction services in recent years, learning from domestic and international emergency disaster reduction experience, constructed a system architecture for integrated and integrated disaster reduction intelligent services, clear integration, integration, key issues in intelligent disaster reduction systems, the key technology of integrated intelligent service system for disaster reduction is put forward, and the first phase platform of the integrated disaster reduction intelligent service system was developed. Finally, the prototype system and some application examples of integrated disaster reduction intelligent service is introduced, to promote the in-depth development and application of integrated disaster reduction intelligent service technology, comprehensively improve China's emergency mapping and assurance capabilities.

\section{Acknowledgement}

This work was supported by the National Key Research and Development Program of China (2016YFC0803108).

\section{References}

Fan W C, Shan C C, et al. (2017). Public safety and emergency management[M]. Science Press.

Zhu Q, Cao Z Y, Lin H, Xie W, Ding Y L. (2014). Key Technologies of Emergency Surveying and Mapping Service System[J]. Geomatics and Information Science of Wuhan University, 39(5):551-555.

Annelli J F. (2006). The national incident management system: A multi-agency approach to emergency response in the United States of America[J]. Revue scientifique et technique-Office international desépizooties, 25(1): 223.

Faraj S, Xiao Y. (2015). Coordination in fast-response organizations[J]. Management science, 52(8): 1155-1169.

Martinis S, Kersten J, Twele A. (2006). A fully automated TerraSAR-X based flood service[J]. ISPRS Journal of Photogrammetry and Remote Sensing, 104: 203-212.

Ajmar A, Boccardo P, Disabato F, et al. (2015). apid Mapping: geomatics role and research opportunities[J]. Rendiconti Lincei, 26(1): 63-73.

Gasparini P, Manfredi G, Zschau J. (2007). Earthquake early warning systems[M]. Berlin: Springer.

Allen R M, Gasparini P, Kamigaichi O, et al. (2009). The status of earthquake early warning around the world: An introductory overview $[\mathrm{J}]$. Seismological Research Letters, 80(5): 682-693.

Jang M, Suh S T. (2010). U-city: new trends of urban planning in Korea based on pervasive and ubiquitous geotechnology and geoinformation[C]//International Conference on Computational Science and Its Applications. Springer, Berlin, Heidelberg, 2010: 262270.

Yu H, Wang J, Wang B, Han H, Chang G. (2017) Generalized total Kalman filter algorithm of nonlinear dynamic errors-in-variables model with application on indoor mobile robot positioning. Acta Geodaetica et Geophysica. DOI :10.1007/s40328-017-0207-7.

Liu M, Zhu J, Zhu Q, et al. (2017) Optimization of simulation and visualization analysis of dam-failure flood disaster for diverse computing systems[J]. International Journal of Geographical Information Science, 31:1-16.

Zhang H, Zhu J, Zhu Q, et al. (2017) A template-based knowledge reuse method for generating multitype 3D railway scenes[J]. International Journal of Digital Earth, 2017: 1-16.

Qiu L, Du Z, Zhu Q, et al. (2017) An integrated flood management system based on linking environmental 
models and disaster-related data[J]. Environmental Modelling \& Software, 91: 111-126.

GUO Renzhong, YING Shen. (2017) The Rejuvenation of Cartography in ICT Era[J]. Acta Geodaetica et Cartographica Sinica, 46(10): 1274-1283.

Li Z, Wang F, Zheng X, et al. (2017) GIS Based Dynamic Modeling of Fire Spread with Heterogeneous Cellular Automation Model and Standardized Emergency Management Protocol [C]. the 3rd ACM SIGSPATIAL International Workshop on the Use of GIS in Emergency Management (EM-GIS 2017).
Wang Y D, Li H, Wang T, Zhu J Q, et al. (2016) The Mining and Analysis of Emergency Information in Sudden Events Based on Social Media. Geomatics and Information Science of Wuhan University, 41(3):290297.

Zhang X X, Bi W W, Yuan Y H. (2015) Study on top level design of informatization construction for disaster prevention and mitigation[J]. China Safety Science Journal, 25(3):159-164. 